\title{
Evaluating the Effects of Bisphenols F and S with Respect to Bisphenol A on Primordial Germ Cell Migration in Zebrafish (Danio rerio) Embryos Using Immunofluorescence Microscopy
}

\author{
Siti Sarah Safura*\#, George Roba*, \& Edward Freeman \\ Department of Biology, St. John Fisher College, NY \\ https:/ / doi.org/10.33697/ajur.2019.031 \\ \#both of these authors contributed equally to this work \\ Students:sss03429@sjfc.edu* and gr00103@siff.edu \\ Mentor:efreeman@sjfc.edu
}

\begin{abstract}
Primordial Germ Cell (PGC) migration occurs in early embryonic development and is highly conserved across taxa. PGC migration occurs within the first 24 hours post fertilization (hpf) in zebrafish, making the organism an efficient model for observing the migration pathway. Proper PGC migration is necessary for normal gonad development and, in some species, sex determination. Disruption of this process leads to defects in gonad formation and abnormal sex determination and differentiation. Studies show that endocrine-disrupting chemicals such as bisphenol A (BPA) disrupt PGC migration in zebrafish. BPA is an estrogenic compound that has been linked to a variety of human diseases, including various cancers, diabetes, reproductive disorders, obesity, and cardiovascular diseases. It is one of the most widely used synthetic compounds worldwide, as it is used to make polycarbonate plastics. Many studies provide evidence of the harmful effects of BPA on living organisms. In response, manufacturers have started to use replacements such as bisphenol F (BPF) and bisphenol S (BPS). However, due to their structural similarity, it is likely that BPF and BPS are just as harmful to organisms as BPA. In this study, we use antibody staining and immunofluorescence microscopy to confirm that BPA exposure results in abnormal PGC migration in zebrafish embryos, as previously studied, and to illustrate that BPF and BPS exposure results in similar PGC migration defects.
\end{abstract}

\section{KEYWORDS}

Zebrafish; Zebrafish Embryos; Primordial Germ Cells; PGC Migration; Gonad Development; Endocrine-Disrupting Chemicals; Bisphenol A; Bisphenol S; Bisphenol F; Sex Determination

\section{INTRODUCTION}

Primordial germ cell migration and sex determination in zebrafish

Primordial germ cells (PGCs) are precursor cells that eventually develop into gametes in sexually-reproducing organisms. PGC migration is a process common among vertebrates and invertebrates, including the common model organisms Xenopus, Drosophila, and Danio rerio. A study comparing mechanisms of PGC migration in Mus musculus, Danio rerio, and Drosophila melanogaster found that although there is variation among the species, it appears that some molecular mechanisms are conserved for PGC migration and maintenance. ${ }^{1} \mathrm{~A}$ number of genes involved in PGC migration are conserved across phyla, indicating that the process is similar in different organisms including those indicated above. ${ }^{2}$ Early in vertebrate development, PGCs migrate from the primary ectoderm to the gonadal ridge where they later develop into gametes. PGC migration is directed by chemical cues secreted by somatic cells. The process is tightly regulated as the pathway that PGCs use to travel to the gonad goes through different tissues. ${ }^{3}$ Migration occurs between embryonic weeks 4 and 6 in humans, ${ }^{4}$ between embryonic days 8 and 10 in mice, ${ }^{5}$ and within 24 hpf (hours post fertilization) in zebrafish. ${ }^{6}$ As zebrafish do not have specific sex chromosomes, sex determination in zebrafish is likely the result of gonadal gene expression, environmental factors, and the presence of PGCs at the gonadal ridge. Therefore, PGC migration and their presence at the gonadal ridge are an important factor in sex determination and eventual differentiation in zebrafish. Defects in the PGC migration pathway have been shown to have adverse effects in zebrafish, including improper gonad formation and infertility. ${ }^{7,8}$ Vasa, an RNA binding protein, is used as a marker for germ cells due to its role in germ cell determination and function. ${ }^{3}$ By analyzing PGCs in zebrafish fixed at $24 \mathrm{hpf}$, we can see a baseline of where germ cells are normally localized and compare this to when embryos are treated with a particular bisphenol. 
Bisphenols in the environment

Bisphenols are synthetic plasticizers that are used in the production of polycarbonate plastics. Bisphenol A (BPA) is a known endocrine-disrupting chemical (EDC) whose estrogenic effects were first observed in 1936.9 In the 1950s, it was found that BPA could be used to make polycarbonate plastics and it became one of the most widely used synthetic compounds worldwide, despite its estrogenic effects. ${ }^{9}$ Bisphenols have been linked to a variety of human diseases, including various cancers, diabetes, reproductive disorders, obesity, and cardiovascular diseases. ${ }^{9}$ The greatest source of human exposure to BPA is leaching of the chemical from everyday household items, including food and beverage containers, thermal receipt paper, and medical devices.,10 Although BPA is biodegradable, trace amounts of BPA are found even in treated wastewater and background concentrations exist in the aquatic environment. ${ }^{11}$

\section{Effects of endocrine-disrupting chemicals (EDCs) on organisms}

Organisms are much more vulnerable to the adverse effects of EDCs in-utero and during the early stages of development, as early developmental processes are tightly regulated by many factors including chemical signaling. Exposure to BPA (an estrogen mimic) during development has been found to lead to reproductive disorders, birth defects, and brain malformations in mammals such as mice and humans. ${ }^{910}$ Human fetal testis tissue, particularly Leydig cells, have been reported to be a major target of BPA. ${ }^{9}$ Concentrations as low as $10 \mathrm{nM}$ were shown to reduce human fetal Leydig cell function, supporting that BPA has estrogenic effects on organ systems and that embryonic exposure is problematic. ${ }^{9}$ Zebrafish share many developmental processes with humans and can therefore provide insight into the potential effects of EDCs in humans. Prior studies have demonstrated that exposure to BPA at doses as low as $0.001 \mu \mathrm{M}$ negatively impact zebrafish reproductive health. ${ }^{12}$ In 2017 , researchers found that higher levels of BPA exposure in zebrafish can cause a reduction in egg production and reduce chances of fertilization. ${ }^{12}$ Because BPA exhibits a non-monotonic dose-response curve, trace BPA concentrations in aquatic environments can still have adverse effects on aquatic organisms. ${ }^{9}$

Due to potential health concerns, Canada (2009), USA (2010), and the European Union (2011) prohibited the use of BPA in the manufacture of polycarbonate feeding bottles for infants. ${ }^{10}$ That being said, the Food and Drug Administration (FDA) has only restricted the use of BPA in baby bottles, sippy cups, and packaging for infant formula. Therefore, consumer concern has pushed companies to replace BPA in other products--it is often replaced with other bisphenols, such as bisphenol F (BPF) and bisphenol $\mathrm{S}$ (BPS). Bisphenols share structural similarities, indicating that they may have similar estrogenic effects in organisms (Figure 1).
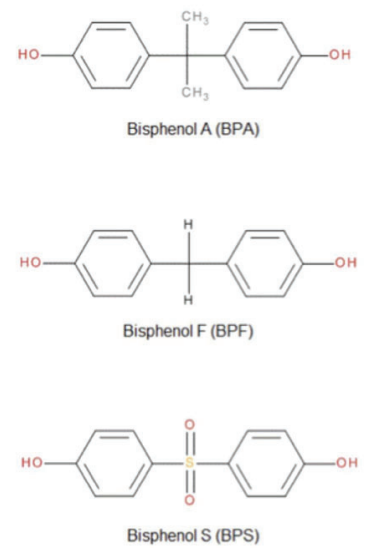

Figure 1. Structures of bisphenols A, F, and S.

All three bisphenols in this study (BPA, BPF, and BPS) are shown to have neurological, metabolic, endocrine, and reproductive effects on organisms. A 2018 study showed that exposure to BPA disrupts differentiation of human-derived neural progenitor cells. ${ }^{13}$ In regards to BPS, exposure to the chemical during development causes precocious neurogenesis in the zebrafish hypothalamus and is associated with hyperactive behavior in zebrafish. ${ }^{14}$ Another study found that exposure to BPF during development affects gonadotropin-releasing hormone neurons in zebrafish through an estrogen-mediated pathway. ${ }^{15} \mathrm{BPA}, \mathrm{BPF}$ and BPS were all found to affect the transcription levels of genes for neurotransmitter-metabolizing enzymes in female rats when exposed during development. ${ }^{16}$ In regards to metabolism, studies show that all three bisphenols are associated with obesity in some context. In a 2019 study, perinatal exposure to BPA in male rats showed that BPA acts as an obesogen as it induced obesity and increased food intake. ${ }^{17}$ There is evidence that BPA exposure during critical periods of development can cause epigenetic changes that are related to the onset of obesity. ${ }^{18}$ The estrogenic potency of BPF and BPS are both in the same order of 
magnitude as BPA and all three bisphenols show the same amount of inhibitory hormonal signaling in adipocytes. ${ }^{19}$ Another study found that both BPS and BPA enhance adipocyte differentiation, and that BPS (a common BPA replacement) is an even stronger adipogen than BPA. ${ }^{20} \mathrm{BPF}$ is also associated with higher risk of obesity in children and adolescents. ${ }^{21}$ These bisphenols are known to have endocrine effects as well: a 2017 study found that all three transactivate estrogen receptors (ERs) in zebrafish. ${ }^{22}$ BPS also alters estradiol-induced non-genomic signaling in rat pituitary cells; this disrupted signaling may result in cell death.23 A 2016 study using both in-vivo and in-vitro studies showed that BPA, BPS, and BPF all upregulate Aromatase B, a key enzyme in estrogen synthesis, in the brains of developing zebrafish. ${ }^{10}$ In a study analyzing the effects of BPA, BPF, and BPS on gene expression in fetal Leydig cells, all three were found to reduce the expression of key genes in testosterone synthesis, further illustrating the estrogenic mimicry of these chemicals. ${ }^{9}$ Another study showed that when adult zebrafish were exposed to 0.002 , 0.02 , and $0.2 \mu \mathrm{M}$ BPS, maternal exposure to BPS greater than $0.02 \mu \mathrm{M}$ caused delayed egg hatching and developmental delays in offspring. ${ }^{24}$ Lastly, a 2013 study showed that exposure to BPA during the first 24 hpf results in abnormal PGC migration in zebrafish embryos. ${ }^{3}$ The current study illustrates that exposure to BPS and BPF also results in abnormal PGC migration in zebrafish.

\section{Bisphenol $A$ and Danio rerio primordial germ cell migration}

Fish are particularly sensitive to the presence of EDCs, due to the environmental and epigenetic cues influencing sex determination and differentiation. ${ }^{25}$ Exposure of zebrafish embryos to 17-alpha-ethinylestradiol ( $\left.\mathrm{EE}_{2}\right)$ showed that estrogen receptors play a role in PGC migration. ${ }^{26}$ Bisphenols are especially of concern, as their wide commercial use causes them to be ubiquitous in the environment and they operate as sex hormone mimics that can alter essential developmental processes. ${ }^{11,14,27} \mathrm{~A}$ 2013 study looked at the effects of BPA on PGC migration in zebrafish and found that BPA exposure during the first 24 hpf at concentrations of $17.5 \mu \mathrm{M}$ and $35 \mu \mathrm{M}$ caused an increased number of PGCs compared to controls and caused PGCs to migrate to ectopic locations. ${ }^{3}$ Failure of PGCs to reach the gonadal ridge leads to defects in sex determination and gonadal differentiation.

Different geographical regions have various amounts of BPA in the environment. Areas near waste treatment plants have varying amounts of BPA, from not detectable to $1.6 \mu \mathrm{M}$ in some, to maximum amounts of $36.8 \mu \mathrm{M}$ and $45.1 \mu \mathrm{M}$ in others, while tap water was found to have a maximum concentration of $5.7 \mu \mathrm{M} .{ }^{28,29,30}$ That being said, because BPA exhibits a non-monotonic dose-response curve, the relationship between dosage and effect may be nonlinear. ${ }^{9}$ In this study, we exposed embryos to published concentrations of BPA $(17.5 \mu \mathrm{M}$ and $35 \mu \mathrm{M})$ that are known to cause abnormal PGC migration in zebrafish during the first $24 \mathrm{hpf}^{3}$ to act as a positive control, and to ensure that fluorescent immunocytochemistry is an effective method for visualizing PGC migration in zebrafish. We exposed embryos to an intermediate dose of BPF and BPS to evaluate whether or not these replacement bisphenols have similar effects on PGC migration as BPA. $25 \mu \mathrm{M}$ was chosen as the intermediate dosage between two known BPA doses that disrupt PGC migration in zebrafish (17.5 $\mu \mathrm{M}$ and $35 \mu \mathrm{M}) .{ }^{3}$ For both BPF and BPS-treated embryos, we expected to see migration patterns similar to those of BPA at similar doses.

\section{METHODS AND PROCEDURES}

\section{Fish maintenance}

Zebrafish were purchased from local pet stores throughout Rochester, NY. Zebrafish were introduced to the housing system and allowed to acclimate to the water and room conditions for two weeks. Experimentation was reviewed and approved by the Institutional Animal Care and Use Committee at St. John Fisher College (IACUC Protocol \#61). Females and males were kept together in tanks that had a constant flow of system water. System water was maintained at $27-29^{\circ} \mathrm{C}$ with a salinity between 1400 1600 microsiemens, which was checked every other day. The $\mathrm{pH}$ level was kept between 6.9-7.4 and was measured twice per week. The fish were kept on a 14 hour light/10 hour dark schedule and fed twice daily. The fish were fed with live brine shrimp (Utah Red Shrimp from Artemac L.L.C) and either Zebrafish select diet (Aquaneering) or flake food (Ocean Star International freshwater aquarium flake food).

\section{Breeding and embryo collection}

Zebrafish embryo collection chambers were created using pipette boxes, with yarn attached to the holes in the top of the box, simulating aquatic plants. The holes allowed embryos to fall into the chamber so they were not consumed by adults. Collection chambers were added to tanks the night prior to collection. After giving the zebrafish one hour for mating, collection chambers were removed from the tanks. Embryos were sorted and dead or unhealthy embryos were then removed.

\section{Endocrine-disrupting chemical exposure}

Within the first hour after fertilization, 3-5 embryos were added to each well of a 24-well plate. Embryos were split into five different treatment groups throughout six different experiments. All embryos were incubated with $998 \mu \mathrm{L}$ of system water and 2 $\mu \mathrm{L}$ of either ethanol or an EDC. Vehicle control embryos were exposed to $2 \mu \mathrm{L}$ of ethanol. BPA, BPF, and BPS groups were exposed to $2 \mu \mathrm{L}$ of each chemical, respectively. Final concentrations were low dose BPA-treated (17.5 $\mu \mathrm{M}$ in ethanol), high dose 
BPA-treated ( $35 \mu \mathrm{M}$ in ethanol), BPF-treated (25 $\mu \mathrm{M}$ in ethanol), and BPS-treated ( $25 \mu \mathrm{M}$ in ethanol). All stock solutions were made in ethanol and stored in the dark to protect from light degradation. The embryos were allowed to grow for 24 hours at 28 ${ }^{\circ} \mathrm{C}$. After 24 hours, if more than two embryos were dead in any well, the embryos in that well were not used for analysis of PGC migration.

\section{Fluorescent microscopy}

After EDC or vehicle treatment for the first $24 \mathrm{hpf}$, embryos were then transferred to $1 \mathrm{~mL}$ centrifuge tubes. Bisphenol solutions were removed and replaced with $4 \%$ paraformaldehyde (PFA). Embryos were then kept in a refrigerator at $4{ }^{\circ} \mathrm{C}$. Embryos were washed the following day with 100\% methanol and placed in $100 \%$ methanol at $-20^{\circ} \mathrm{C}$ for long term storage. Before staining, embryos were dechorionated using surgical tweezers and a Leica dissecting microscope (\#S8APO). The staining procedure began with three 30 minute $500 \mu \mathrm{L}$ washes in a phosphate-buffered saline (PBS) $/ 1 \%$ triton X-100 solution at room temperature. Next, embryos received two one-hour washes and two 5 minute washes, each using $500 \mu \mathrm{L}$ of blocking buffer containing PBS/1\% triton X-100 with 10\% goat serum. Embryos were then placed in a solution containing $2 \mu \mathrm{L}$ of primary antibody (rabbit anti-vasa, Abcam \#ab209710) added to $298 \mu \mathrm{L}$ of blocking buffer (1:150 dilution). Primary antibody incubations were done overnight with gentle rotation on an orbital shaker at $4{ }^{\circ} \mathrm{C}$. Following the primary antibody incubations, embryos underwent three one-hour washes in $500 \mu \mathrm{L}$ of blocking buffer at room temperature. Next, embryos received six 10 minute washes with PBS/1\% triton X100 at room temperature. Finally, $3 \mu \mathrm{L}$ of secondary antibody (goat-anti-rabbit IgG-FITC, Santa Cruz \# sc-2012) were added to $297 \mu \mathrm{L}$ of blocking buffer (1:100 dilution) for incubation with the embryos. Secondary antibody incubations were done overnight with gentle rotation on an orbital shaker at $4{ }^{\circ} \mathrm{C}$. Following the secondary antibody incubations, embryos underwent three 10 minute washes with $500 \mu \mathrm{L}$ of PBS/1\% triton X-100 at room temperature. Embryos were then placed onto concave slides with minimal buffer and visualized using a Leica fluorescent microscope while avoiding light exposure to prevent fading of FITC. Embryos were visualized with a 10X objective and photographs were taken using the Q-Capture Pro 7 program (Figure 2).

Data and Statistical Analysis

After visualization, the embryos were categorized into either normal (clustered) or abnormal (dispersed) groups for statistical analysis (Table 1). Through the use of Social Science Statistics software, Fisher's Exact tests were ran on experimental groups to see if they were significantly different from control, as well as to see if there was a significant difference between bisphenols (Table 2).

\section{RESULTS}

Control embryos visualized under the microscope showed a clustered localization pattern, as expected (Figure 2, A and B). For the purposes of this study, "dispersed localization" was defined as any localization of PGCs significantly different from control. These embryos demonstrated a non-clustered pattern with visible spacing between cells. Embryos were categorized into either normal (clustered at the gonadal ridge) or abnormal (dispersed). After visualization, specific distances were not quantified. Fluorescent microscopy with BPF and BPS-treated embryos showed a similar PGC staining pattern as BPA-treated embryos, which served as a positive control (Figure 2). The majority of bisphenol-treated embryos showed dispersed PGC localization when compared to control (Table 1). Percentages of dispersed localization are also represented graphically (Figure 3). BPA, which is known to cause dispersed PGC migration, served as our positive control and was also compared to BPS and BPF-treated groups (Table 2). 


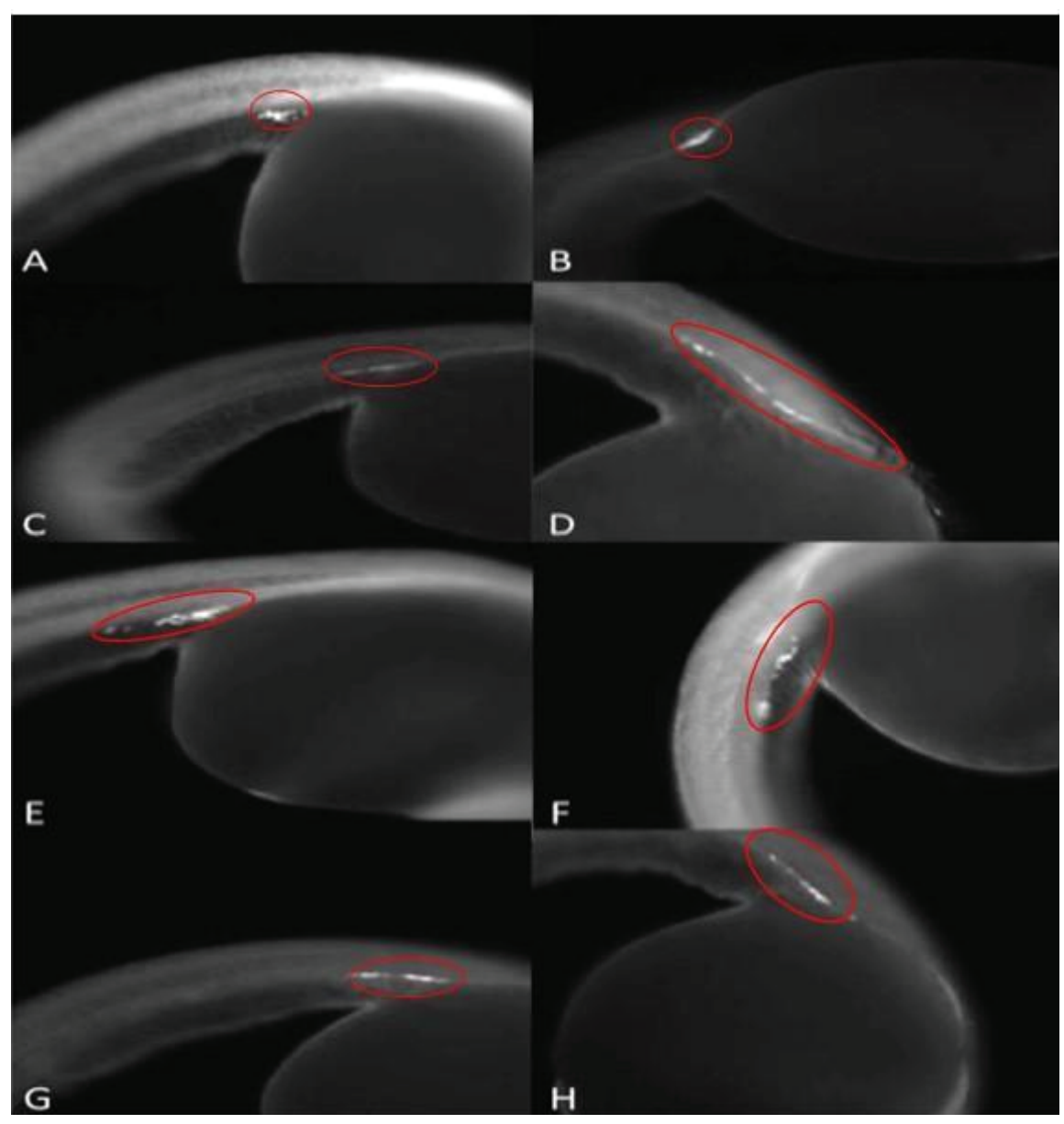

Figure 2. BPA, F, and S representative staining patterns. A and B) Control embryos, C) $17 \mu \mathrm{M}$ BPA-treated, D) $35 \mu \mathrm{M} \mathrm{BPA}$-treated, E and F) $25 \mu \mathrm{M}$ BPF-treated, $\mathrm{G}$ and H) $25 \mu \mathrm{M}$ BPS-treated.

\begin{tabular}{|c|c|c|}
\hline Treatment & Number of embryos & Dispersed migration (\%) \\
\hline Vehicle Control & 31 & $2 / 31(6 \%)$ \\
\hline Positive Control: $17.5 \mu \mathrm{M} \mathrm{BPA}$ & 38 & $31 / 38(81.5 \%)$ \\
\hline Positive Control: $35 \mu \mathrm{M} \mathrm{BPA}$ & 31 & $31 / 31(100 \%)$ \\
\hline $25 \mu \mathrm{M} \mathrm{BPF}$ & 32 & $26 / 32(81 \%)$ \\
\hline $25 \mu \mathrm{M}$ BPS & 32 & $29 / 32(91 \%)$ \\
\hline
\end{tabular}

Table 1. Treatments, number of embryos per treatment, and percentage of embryos with dispersed migration. Data are representative of six experiments using fluorescent immunocytochemistry. 


\section{Percentage of Embryos with Dispersed PGC Migration}

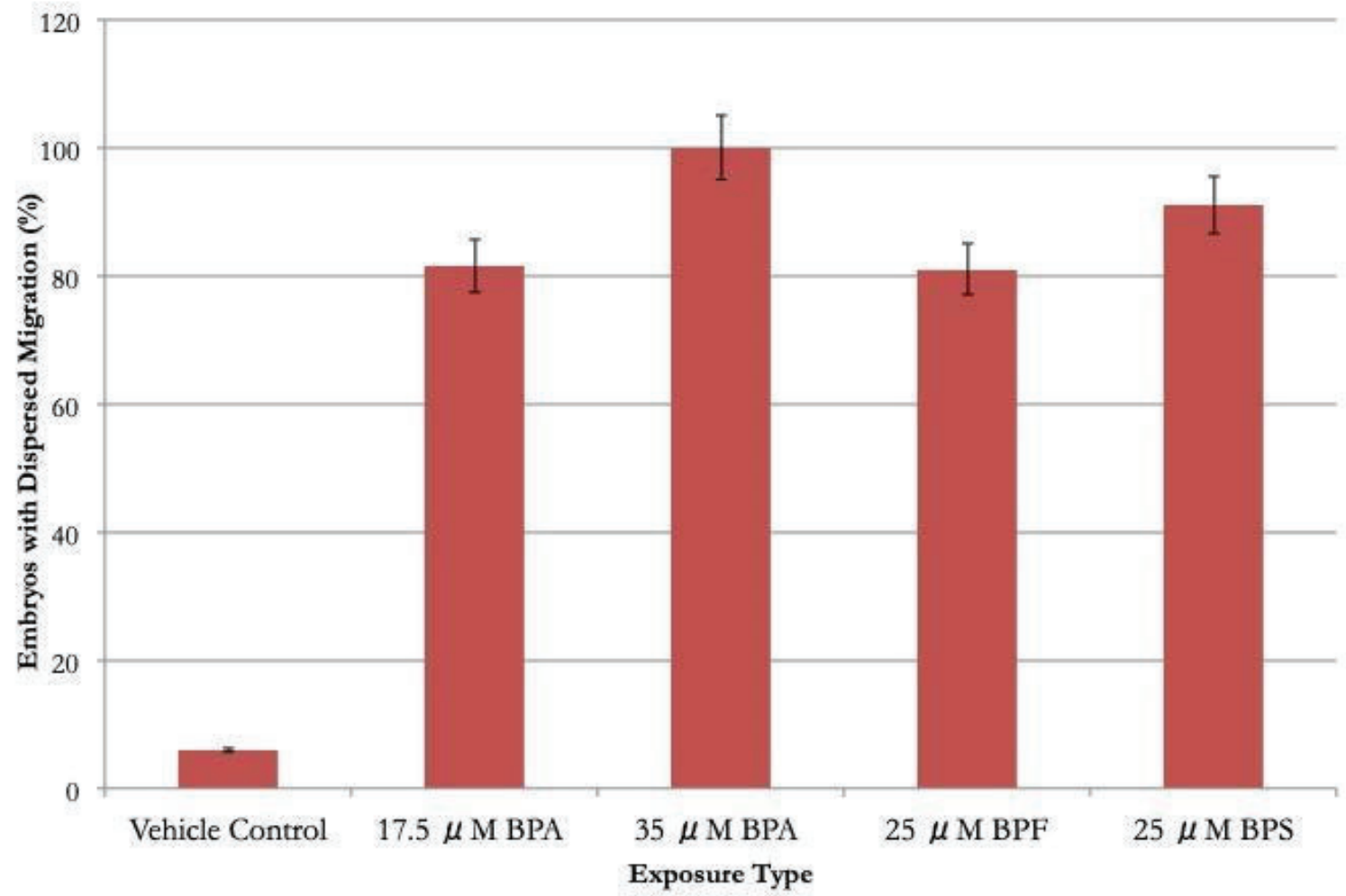

Figure 3. Percentage of embryos with dispersed migration in each experimental group.

\begin{tabular}{|c|c|c|c|c|c|}
\hline & Vehicle Control & $17.5 \mu \mathbf{M}$ BPA & $35 \mu \mathbf{M ~ B P A}$ & $25 \mu \mathbf{M}$ BPF & $25 \mu \mathbf{M}$ BPS \\
\hline $25 \mu \mathbf{M}$ BPF & SS & NS & NS & -- & NS \\
\hline $25 \mu$ MBPS & SS & NS & NS & NS & -- \\
\hline $17.5 \mu$ M BPA & SS & -- & NS & NS & NS \\
\hline $35 \mu$ M BPA & SS & NS & -- & NS & NS \\
\hline
\end{tabular}

Table 2. Statistical significance of differences between groups. Fisher's Exact tests were done between treatment groups. "NS" indicates the difference is not statistically significant. "SS" indicates the difference is statistically significant. A dash indicates that the test was not done. All tests were done using p-value $<0.01$.

Data and statistical analysis showed that the differences between all bisphenol-exposed groups and vehicle control were statistically significant (Table 2). No statistically significant difference was found when comparing bisphenol-exposed groups to each other, illustrating that BPA, BPS, and BPF all have similar effects on PGC migration in zebrafish embryos.

\section{DISCUSSION}

The existence of EDCs in the environment is the result of human activity, as EDCs are not produced naturally. The ecological implications of releasing bisphenols into the environment are not yet fully understood. Bisphenols A, F, and S are known to disrupt a variety of processes in many organisms. A 2014 study found that embryonic BPS exposure at 0.004, 0.04, and 0.4 $\mu \mathrm{M}$ 
caused zebrafish sex ratios to be skewed towards females. ${ }^{31}$ Another study found that germline-deficient zebrafish developed as phenotypic males without germ cells and were therefore unable to reproduce. ${ }^{7}$ Based on this information, it is possible that wild populations would see skewed sex ratios and infertility from bisphenol exposure, hurting the species' chances of survivalespecially in the presence of other selective pressures. A 2013 study found that doses of BPA 1-4 magnitudes of order lower than a commonly used lowest observed adverse effect level (LOAEL) tested in traditional toxicology assessments of $50 \mathrm{mg} / \mathrm{kg} / \mathrm{day}$ can have adverse effects on animals. ${ }^{32}$ It is logical to predict that due to their structural similarities, BPA replacements BPF and BPS may have similar low dose effects. Not only does this have ecological implications, but it likely affects human health as well, since manufacturers continue to use bisphenols. Further research on the effects and mechanisms of these EDCs would contribute to the literature that can be used to persuade lawmakers to enforce the use of safer alternatives, to reduce environmental and consumer exposure, and to create more effective waste management policies.

The mechanism of EDC action that disrupts PGC migration is not understood and could involve many different parameters within either the PGCs, the somatic cells of the gonad, or both. It is likely that aromatase plays a key role in the mechanism of action of bisphenols, as this is the key enzyme in estrogen synthesis. In a 2014 study, simultaneous exposure to BPA and fadrozole (an aromatase inhibitor) showed inhibition of BPA-induced effects. ${ }^{14}$ This may indicate that BPA requires aromatase in order to cause abnormalities. Furthermore, a 2016 study that looked at the effects of inhibiting aromatase alone found that the manipulation of the aromatase system during the critical period of sexual differentiation is responsible for a complete and irreversible alteration of the process of gonadal differentiation in zebrafish. ${ }^{33}$ Therefore, aromatase plays an essential role in sexual differentiation in zebrafish and may be a target of bisphenols in inducing PGC migration defects.

\section{CONCLUSIONS}

We report that bisphenols $\mathrm{F}$ and $\mathrm{S}$ cause abnormal PGC migration patterns in zebrafish, similar to those of BPA. After conducting antibody staining and fluorescent microscopy on BPF and BPS-treated embryos, it appears that neither BPF nor BPS are suitable replacements for BPA, as they all disrupt PGC migration at similar exposure levels. BPA, BPF, and BPS are all compounds with adjoined phenolic rings, differing in the identity of the functional group connecting the two rings (Figure 1). It is likely that the phenolic rings, common among the three different compounds, have a similar biochemical function. However, the exact mechanism by which bisphenols cause these effects is unknown. It is important to note that bisphenols are not the only endocrine-disrupting chemicals that affect PGC migration. Embryonic exposure to estrogenic EDCs nonylphenol and endosulfan also disrupted normal PGC migration. ${ }^{34}$ It is likely that the chemical cues secreted from somatic cells, that direct PGC migration, are interrupted by nonylphenol and endosulfan. ${ }^{34}$ Bisphenol exposure may also interrupt this signaling, causing abnormal migration.

Insight on the mechanism of BPA and its analogues would be useful in learning how to provide safe alternatives to these plasticizers. Goals for expanding on our results include examining other bisphenols to study how their functional groups relate to PGC migration. Bisphenol $G$ is of particular interest due to its additional functional groups attached to the two trademark phenolic rings found in most bisphenols. Bisphenol BP is also of interest because it has two extra phenolic rings. Knowing if small differences in chemical structure cause significant differences in effects on organisms could also help find safer alternatives to BPA. The use of fadrozole, an aromatase inhibitor, has been utilized to prevent the activity of BPA in various systems and its use in preventing altered PGC migration could also be explored. Finally, decreasing to nanomolar exposure concentrations would help to determine the point of effect drop-off.

It is also important to consider that in the environment, exposure to EDCs is variable. It is unlikely that organisms are exposed to a constant amount of a single EDC for a fixed amount of time; therefore, it is likely that organisms have a time period in which they can recover from exposure. ${ }^{32} \mathrm{Few}$ studies evaluate the ability of zebrafish to recover from EDC-induced effects, therefore more research could be done to see if the effects of certain estrogenic EDCs are reversible.

\section{ACKNOWLEDGMENTS}

Authors thank Jennifer Gantress, Lab Manager, for purchasing supplies and St. John Fisher College for providing budgetary support. 


\section{REFERENCES}

1. Richardson, B.E. and Lehmann, R. (2010) Mechanisms guiding primordial germ cell migration: strategies from different organisms, Nat Rev Mol Cell Biol 11(1), 37-49. https:/ / doi.org/10.1038/nrm2815

2. Saito, T., Goto-Kazeto, R., Kawakami, Y., Nomura, K., Tanaka, H., Adachi, S., Arai, K., and Yamaha, E. (2011) The mechanism for primordial germ cell migration is conserved between Japanese eel and zebrafish, PLoS ONE, 6(9), e24460. bttps:/ / doi.org/10.1371/journal.pone.0024460

3. Akbulut, C., Kizil, A., and Yon, N.D. (2013) Effects of low doses of bisphenol A on primordial germ cells in zebrafish (Danio rerio) embryos and larvae, Kafkas Universitesi Veteriner Fakuittesi Dergisi, 19(4), 647-653. https:/ / doi.org/10.9775/ kvfd.2013.8600

4. Fujimoto, T., Miyayama, Y., and Fuyuta, M. (1977) The origin, migration and fine morphology of human primordial germ cells, Anat Rec, 188(3), 315-329. https:/ / doi.org/10.1002/ar.1091880305

5. Wear, H.M., McPike, M., and Watanabe, K. (2016) From primordial germ cells to primordial follicles: a review and visual representation of early ovarian development in mice, J Ovarian Res, 9: 36. bttps:// doi.org/10.1186/s13048-016-0246-7

6. Paksa, A. and Raz, E. (2015) Zebrafish germ cells: motility and guided migration, Curr Opin Cell Biol, $36,80-85$. bttps:/ / doi.org/10.1016/j.ceb.2015.07.007

7. Siegfried, K.R. and Nüsslein-Volhard, C. (2008) Germ line control of female sex determination in zebrafish, Dev Biol, 324(2), 277-287. https:// doi.org/10.1016/j.ydbio.2008.09.025

8. Wong, T.T. and Collodi, P. (2013) Inducible sterilization of zebrafish by disruption of primordial germ cell migration, PLoS ONE, 8(6): e68455. https:// doi.org/10.1371/journal.pone.0068455

9. Eladak, S., Grisin, T., Moison, D., Guerquin, M.J., N’Tumba-Byn, T., Pozzi-Gaudin, S., Benachi, A., Livera, G., RouillerFabre, V., and Habert, R. (2015) A new chapter in the bisphenol A story: bisphenol S and bisphenol F are not safe alternatives to this compound, Fertil Steril, 103(1), 11-21. https:/ / doi.org/10.1016/j.fertnstert.2014.11.005

10. Cano-Nicolau, J., Vaillant, C., Pellegrini, E., Charlier, T.D., Kah, O., and Coumailleau, P. (2016) Estrogenic effects of several BPA analogs in the developing zebrafish brain, Front Neurosci, 10, 112. https:// doi.org/10.3389/fnins.2016.00112

11. Tsai, W. T. (2006) Human health risk on environmental exposure to bisphenol-A: a review, J Environ Sci Health C Environ Carcinog Ecotoxicol Rev, 24(2), 225-255. https:/ / doi.org/10.1080/10590500600936482

12. Chen, J., Saili, K.S., Liu, Y., Li, L., Zhao, Y., Bai, C., Tanguay, R.L., Dong, Q., and Huang, C. (2017) Developmental bisphenol A exposure impairs sperm function and reproduction in zebrafish, Chemosphere,169, 262-270. https:/ / doi.org/ 10.1016/j.chemosphere.2016.11.089

13. Fujiwara, Y., Miyazaki, W., Koibuchi, N., and Katoh, T. (2018) The effects of low-dose bisphenol A and bisphenol F on neural differentiation of a fetal brain-derived neural progenitor cell line, Frontiers in Endocrinology, 9, 1-9. bttps:// doi.org/10.3389/fendo.2018.00024

14. Kinch, C.D., Ibhazehiebo, K., Jeong, J.H., Habibi, H.R., and Kurrasch, D.M. (2014) Low-dose exposure to bisphenol A and replacement bisphenol S induces precocious hypothalamic neurogenesis in embryonic zebrafish, Proc Natl Acad Sci USA, 112(5), 1475-1480. https:/ / doi.org/10.1073/pnas.1417731112

15. Weiler, K. and Ramakrishnan, S. (2019) Bisphenol F causes disruption of gonadotropin-releasing hormone neural development in zebrafish via an estrogenic mechanism, NeuroToxicology, 71, 31-38. https:// doi.org/10.1016/j.neuro.2018.12.001

16. Castro, B., Sanchez, P., Torres, J.M., and Ortega, E. (2015) Bisphenol A, bisphenol F, and bisphenol S affect differently 5areductase expression and dopamine-serotonin systems in the prefrontal cortex of juvenile female rats, Environ Res, 142, 281287. https:// doi.org/10.1016/j.envres.2015.07.001

17. Stoker, C., Andreoli, M.F., Kass, L., Bosquiazzo, V.L., Rossetti, M.F., Canesini, G., Luque E.H., and Ramos, J.G. (2019) Perinatal exposure to bisphenol A (BPA) impairs neuroendocrine mechanisms regulating food intake and kisspetin system in adult male rates. Evidence of metabolic disruptor hypothesis, Mol Cell Endocrinol, 499. https:// doi.org/10.1016/j.mce.2019.110614

18. vom Saal, F.S., Nagel, S.C., Coe, B.L., Angle, B.M., and Taylor, J.A. (2012) The estrogenic endocrine disrupting chemical bisphenol A (BPA) and obesity, Mol Cell Endocrinol, 354(1-2), 74-84. https:/ / doi.org/ 10.1016/j.mce.2012.01.001

19. Rochester, J.R. and Bolden, A.L. (2015) Bisphenol S and F: A systematic review and comparison of the hormonal activity of bisphenol A substitutes, Environ Health Perspect, 123(7), 643-650. bttps:/ / doi.org/ 10.1289/ ehp.1408989

20. Ahmed, S. and Atlas, E. (2016) Bisphenol S and bisphenol A induced adipogenesis of murine preadipocytes occurs through direct peroxisome proliferator-activated receptor gamma activation, Int J Obes, 40, 1566-1573. bttps:/ / doi.org/10.1038/ijo.2016.95

21. Liu, B., Lehmler, H., Sun, Y., Xu, G., Sun, Q., Snetselaar, L.G., Wallace, R.B., and Bao, W. (2019) Association of bisphenol A and its substitutes, bisphenol F and bisphenol S, with obesity in United States children and adolescents, Diabetes Metab J, 43(1), 59-75. https:/ / doi.org/10.4093/dmj.2018.0045

22. Le Fol, V., Aït-Aïssa, S., Sonavane, M., Porcher, J., Balaguer, P., Cravedi, J., Zalko, D., and Brion, F. (2017) In vitro and in vivo estrogenic activity of BPA, BPF, and BPS in zebrafish-specific assays, Ecotox Environ Safe, 142, 150-156.

https://doi.org/10.1016/j.ecoenv.2017.04.009 
23. Viñas, R. and Watson, C.S. (2013) Bisphenol S disrupts estradiol-induced nongenomic signaling in a rat pituitary cell line: effects on cell functions, Environ Health Perspect, 121(3), 352-358. bttps:/ / doi.org/ 10.1289/ ehp.1205826

24. Ji, K., Hong, S., Kho, Y., and Choi, K. (2013) Effects of bisphenol S exposure on endocrine functions and reproduction of zebrafish, Environ Sci Technol, 47(15), 8973-8800. https://doi.org/10.1021/es400329t

25. Nagabhushana, A. and Mishra, R.K. (2016) Finding clues to the riddle of sex determination in zebrafish, J Biosci, 41, $145-155$. bttps:/ / www.ncbi.nlm.nih.gov/pubmed/ 26949096

26. Hu, J., Sun, S., Guo, M., and Song, H. (2014) Use of antagonists and morpholinos in loss-of-function analyses: estrogen receptor ESR2a mediates the effects of 17alpha-ethinylestradiol on primordial germ cell distribution in zebrafish, Reprod Biol Endocrinol, 12:40. https:/ / doi.org/10.1186/1477-7827-12-40

27. Tse, W.K.F., Yeung, B.H.Y., Wan, H.T., and Wong, C.K.C. (2013) Early embryogenesis in zebrafish is affected by bisphenol A exposure, Biol Open, 2(5), 466-471. https:// doi.org/10.1242/ bio.20134283

28. Corrales, J., Kristofco, L.A., Steele, W.B., Yates, B.S., Breed, C.S., Williams, E.S., and Brooks, B.W. (2015) Global assessment of bisphenol A in the environment: review and analysis of its occurrence and bioaccumulation, Dose-Response ,13, 1-29. bttps://doi.org/10.1177/1559325815598308

29. Fukazawa, H., Watanabe, M., Shiraishi, F., Shiraishi, H., Shiozawa, T., Matsushita, H., and Terao, Y. (2002) Formation of chlorinated derivatives of bisphenol A in waste paper recycling plants and their estrogenic activities, J Health Sciences 48, 242249. https:// doi.org/10.1248/jhs.48.242

30. Urase, T. and Miyashita, K. (2003) Factors affecting the concentration of bisphenol A in leachates from solid waste disposal sites and its fate in treatment processes, J Mater Cycles Waste Manag, 77-82. http:/ / dx.doi.org/10.1007/s101630300012

31. Naderi, M., Wong, M., and Gholami, F. (2014) Developmental exposure of zebrafish (Danio rerio) to bisphenol-S impairs subsequent reproduction potential and hormonal balance in adults, Aquat Toxicol, 148, 195-203. bttps:// doi.org/10.1016/j.aquatox.2014.01.009

32. Vandenberg, L.N., Ehrlich, S., Belcher, S.M., Ben-Jonathan, N., Dolinoy, D.C., Hugo, E.R., Hunt, P.A., Newbold, R.R., Rubin, B.S., Saili, K.S., Soto, A.M., Wang, H., and vom Saal, F.S. (2013) Low dose effects of bisphenol A, Endocrine Disruptors, 1:1, e26490. https://doi.org/10.4161/endo.26490

33. Luzio, A., Monteiro, S.M., Rocha, E., Fontaínhas-Fernandes, A.A., and Coimbra, A.M. (2016) Development and recovery of histopathological alterations in the gonads of zebrafish (Danio rerio) after single and combined exposure to endocrine disruptors (17a-ethinylestradiol and fadrozole), Aquat Toxicol, 175, 90-105. bttps:// doi.org/10.1016/j.aquatox.2016.03.014

34. Willey, J.B. and Krone, P.H. (2001) Effects of endosulfan and nonylphenol on the primordial germ cell population in prelarval zebrafish embryos, Aquat Toxicol, 54,(1-2), 113-123. https:// www.ncbi.nlm.nih.gov/pubmed/11451430

\section{ABOUT STUDENT AUTHORS}

Siti Sarah Safura graduated with a BS in Biology with minors in Chemistry and Women \& Gender Studies from St. John Fisher College in 2017 and an MS in Medical Humanities from the University of Rochester in 2018. She is currently pursuing a career in medicine. George Roba graduated with a BS in Biology, a BA in Psychology, and a minor in Spanish for the Health Care Professional from St. John Fisher College in 2018. He has recently completed training as a paramedic and is pursuing a career in healthcare.

\section{PRESS SUMMARY}

This project was done to analyze the effects of BPF and BPS on primordial germ cell (PGC) migration in zebrafish embryos. Zebrafish are a commonly used model organism and are an excellent model for analyzing the effects of EDCs. Proper PGC migration is necessary in vertebrates, including humans, for proper gonad formation. BPA has been shown to cause abnormal PGC migration in zebrafish embryos. BPS and BPF are commercial plasticizers often used as replacements for BPA. Numerous reports have demonstrated the negative effects of BPA exposure on organisms including zebrafish, mice, and humans. However, work describing the impacts of BPF and BPS are still emerging. Because all three bisphenols share structural similarities, it is possible that they have similar effects on PGC migration. This study illustrates that at similar doses to BPA, BPF and BPS cause abnormal PGC migration in zebrafish embryos. 